\title{
Sécurité des produits, respect des normes techniques et conformité aux exigences essentielles: les précisions du Tribunal fédéral
}

\section{Anne-Christine Fornage *}

Le Tribunal fédéral s'est penché sur les exigences essentielles auxquelles un produit doit répondre pour sa mise sur le marché sous l'angle du droit de la sécurité des produits. Le producteur qui démontre avoir respecté des normes techniques harmonisées peut ainsi bénéficier de la présomption que son produit remplit de telles exigences. Il incombe alors aux organes de surveillance du marché d'apporter la preuve que tel n'est pas le cas, ce qu'est précisément parvenu à démontrer la SUVA dans le cas d'espèce. L'auteure saisit l'occasion de cette jurisprudence pour porter un regard critique sur le cadre légal suisse de la mise sur le marché des produits et de leur surveillance.

Introduction 110

I. L'état de fait ....................................................................................... 110

II. Les précisions du Tribunal fédéral et leur commentaire ................................. 111

1. La législation suisse en matière de sécurité des produits .............................112

a) Du droit européen au droit suisse de la sécurité des produits ....................112

b) De la LSPro à la LETC en passant législation sectorielle ...........................114

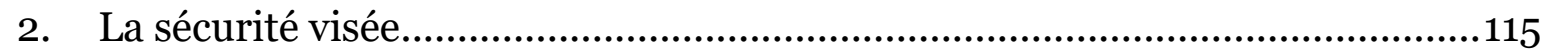

a) Le risque pour la santé et la sécurité …....................................................115

b) Les conséquences pour la surveillance du marché.................................. 118

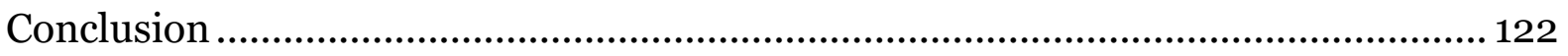

Zitiervorschlag: Anne-Christine Fornage, Sécurité des produits, respect des normes techniques et conformité aux exigences essentielles: les précisions du Tribunal fédéral, in: sui-generis 2018, S. 109

URL: $\quad$ sui-generis.ch/62

DOI: $\quad$ https://doi.org/10.21257/sg.62

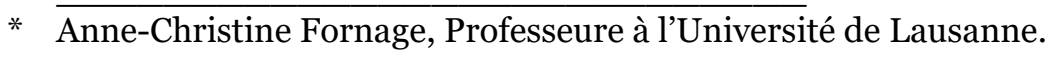

Dieses Werk ist lizenziert unter einer Creative Commons Namensnennung - Weitergabe unter gleichen Bedingungen 4.0 International Lizenz. 


\section{Introduction}

1

Le 10 avril 2017, le Tribunal fédéral a prononcé l'arrêt 2C_75/2016, publié en partie aux ATF 143 II 518. Destinée à la publication, la décision porte sur l'application de la loi fédérale sur la sécurité des produits ${ }^{1}$ (ci-après : LSPro). Il y est plus particulièrement question du respect par le producteur des normes techniques et du rôle de celles-ci par rapport aux exigences essentielles en matière de conformité. Après avoir exposé l'état de fait de l'arrêt (I), nous rapporterons les principales réflexions des juges de Mon-Repos et les commenterons (II).

\section{L'état de fait}

2 A. SA est une entreprise ayant pour but statutaire le commerce, la construction et l'entretien d'engins de construction et de pièces usagées sur des machines de terrassement et de chantier de toutes sortes. A la suite de deux tragiques accidents survenus en juin 2013, en lien avec l'utilisation de systèmes d'attache rapide pour la fixation d'accessoires sur un excavateur fabriqués par A. SA, la Caisse nationale suisse d'assurance en cas d'accidents, en allemand "Schweizerische Unfallversicherungsanstalt » (ciaprès : SUVA), a ouvert une procédure de contrôle.

3 Le 13 mars 2014, la SUVA a prononcé une interdiction de mise sur le marché des dispositifs d'attache rapide, dès le $1^{\mathrm{er}}$ janvier 2016 et aussi longtemps que ceux-ci ne respecteraient pas la Directive 2006/42/CE relative aux machines ${ }^{2}$, en

1

Loi fédérale du 12 juin 2009 sur la sécurité des produits (RS 930.11).

2 Directive 2006/42/CE du Parlement européen et du Conseil du 17 mai 2006 relative aux machines particulier les exigences concernant l'évaluation du risque. Elle a estimé que le respect des normes harmonisées allégeait certes l'examen auquel le fabricant doit s'adonner, sans toutefois lui permettre d'y renoncer. Selon elle, la norme $\mathrm{SN}$ «EN 474-1 » (ci-après : norme «EN 474-1») concrétisait en partie les exigences essentielles de la Directive pour les dispositifs d'attache rapide. Son respect ne permettait cependant pas de conclure que tous les risques étaient couverts. Les dispositifs présentaient ainsi une mise en danger sérieuse provenant d'une combinaison de facteurs, soit le verrouillage incomplet et défectueux des dispositifs d'attache rapide et le comportement fautif du conducteur de la machine. Comme ce comportement était suffisamment prévisible, le fabricant aurait dû prendre des mesures appropriées pour en éviter la survenance. La mise en œuvre de mesures de construction aurait en premier lieu permis de diminuer voire d'éviter la réalisation de ces risques. Des solutions techniques pour les produits en circulation auraient en deuxième lieu pu pour partie être couronnées de succès. Plusieurs accidents mortels ayant eu lieu à l'occasion de l'utilisation des dispositifs d'attache rapide litigieux qui pouvaient être reliés aux défauts constatés auraient pu avec suffisamment de vraisemblance être évités si des mesures techniques avaient été prises pour parer à la chute d'un accessoire des engins de construction.

et modifiant la directive 95/16/CE (refonte), JO L 157 du 9 juin 2006, p. 24 ; modifiée en dernier lieu par la directive 2014/33/UE, JO L 96 du 29 mars 2014, p. 251 (ci-après: Directive 2006/42/CE relative aux machines). 
4 A. SA a fait recours contre cette décision auprès du Tribunal administratif fédéral le 25 avril 2014. Elle a obtenu gain de cause le 9 décembre 2015. Le 25 janvier 2016, la SUVA a saisi le Tribunal fédéral d'un recours de droit public en concluant à l'annulation du jugement du Tribunal administratif et à la confirmation de sa décision du 13 mars 2014, avec la précision que la partie du considérant «et dispositifs d'attache rapide similaires » de sa propre décision ne devait pas être reprise.

5 Le Département fédéral de la formation, de l'économie et de la recherche (ciaprès : DEFR) a également fait recours auprès des juges de Mon-Repos en prenant des conclusions similaires. Le 10 avril 2017, la deuxième Cour de droit public du Tribunal fédéral n'est pas entrée en matière sur l'écriture de la SUVA. Elle a en revanche confirmé la décision rendue précédemment par l'institution en admettant le recours du DEFR avec la modification proposée.

\section{Les précisions du Tribunal fédéral et leur commentaire}

Dans sa décision, le Tribunal fédéral commence par dénier la qualité pour recourir de la SUVA. L'institution, en sa qualité d'établissement de droit public doté de la personnalité juridique conformément à l'art. 61 de la loi fédérale sur l'assurance-accidents 3 , joue en effet un rôle particulier en matière de surveillance du marché. Il s'agit d'un organe désigné par l'ordonnance sur la sécurité des

3 Loi fédérale du 20 mars 1981 sur l'assuranceaccidents (RS 832.20). produits 4 (ci-après : OSPro) pour contrôler l'application des prescriptions de mise sur le marché pour les produits litigieux (art. 20 al. 1 let. a OSPro) et les machines litigieuses (art. 19 let. a OSPro) qui agit conformément aux compétences réglées par le DEFR5.

7 En tant qu'autorité ayant prononcé la première décision d'interdiction dans l'affaire soumise aux juges de Mon-Repos et en vertu de l'art. 89 al. 1 de la loi sur le Tribunal fédéral ${ }^{6}$ (ci-après : LTF), la SUVA n'est en principe pas légitimée à remettre en cause la décision du Tribunal administratif fédéral7. Elle peut certes être concernée dès lors qu'un jugement influe sur l'accomplissement futur d'une tâche publique, mais cet argument ne lui ouvre pas à lui seul le droit de recourir'8. Le Tribunal fédéral réfute en outre le fait qu'elle soit touchée comme une personne privée et rappelle que cette exception s'interprète de manière restrictive 9 . Il admet en revanche la qualité pour recourir du DEFR, en vertu de l'art. 89 al. 2 let. a LTF.

8 L'arrêt prononcé le 10 avril 2017 s'articule autour de deux axes que nous développerons et commenterons successivement. Il s'agit premièrement de la législation suisse en matière de sécurité des produits (1) et secondement de la sé-

$4 \overline{\text { Ordonnance du } 19 \text { mai } 2010 \text { sur la sécurité des }}$ produits (RS 930.111).

5 Cf. l'annexe de l'ordonnance du DEFR du 18 juin 2010 sur l'exécution de la surveillance du marché conformément à la section 5 de l'ordonnance sur la sécurité des produits (RS 930.111.5).

6 Loi sur le Tribunal fédéral du 17 juin 2005 (RS 173.110).

ATF 140 V 521 consid. 2.1.1.

8 Arrêt du Tribunal fédéral 2C_75/2016 du 10 avril 2017, publié en partie aux ATF 143 II 518, consid. 2.3.1.

$9 \quad$ ATF 141 II 161 consid. 2. 
curité visée pour les produits sur le marché, plus particulièrement pour les machines litigieuses (2).

\section{La législation suisse en matière de sécurité des produits}

9 La décision du Tribunal fédéral confirmant l'interdiction de mise sur le marché des machines litigieuses s'appuie sur la législation en matière de sécurité des produits. Elle nous donne l'occasion de retracer les principales étapes de l'adoption du droit suisse en la matière, utiles pour saisir les liens étroits que celui-ci entretient avec le droit européen, omniprésent en ce domaine (1.a) et de préciser l'articulation des différents textes légaux, à savoir principalement la LSPro, la loi fédérale sur les entraves techniques au commerce ${ }^{10}$ (ci-après : LETC) et la législation sectorielle sur la sécurité des produits (1.b).

\section{a) Du droit européen au droit suisse de la sécurité des produits}

10 Le $1^{\text {er }}$ juillet 2010, date de l'entrée en vigueur de la LSPro, marque un tournant dans la législation suisse en matière de sécurité des produits. Auparavant, c'est la loi fédérale sur la sécurité d'installations et d'appareils techniques ${ }^{11}$ (ci-après : LSIT) qui traitait de la sécurité de ces installations et appareils. Son adoption était allée de pair avec la volonté manifestée par le législateur d'œuvrer en faveur de l'élimination des obstacles

10 Loi fédérale sur les entraves techniques au commerce du 6 octobre 1995, révisée en 2010 (RS 946.51).

11 Loi fédérale sur la sécurité d'installations et d'appareils techniques du 19 mars 1976, abrogée

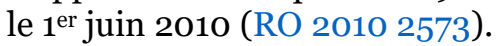

techniques au commerce ${ }^{12}$, la LSIT avait ainsi été adoptée en même temps que la LETC dans sa version antérieure.

11 La LSPro est le résultat d'une reprise autonome du droit européen. Le législateur suisse a en effet transposé la Directive 2001/95/CE relative à la sécurité générale des produits ${ }^{13}$. Cette reprise est intervenue pour renforcer la confiance et la sécurité des produits sur le marché suisse $^{14}$. Elle s'inscrit dans la volonté affichée par la Confédération de tenir compte du droit européen nonobstant le refus du peuple et des cantons d'adhérer à l'Espace Economique Européen. De nombreux actes législatifs d'origine européenne ont de fait trouvé un écho en droit suisse depuis les années 1990, en particulier dans le domaine de la sécurité des produits.

12 On pense d'abord à la "nouvelle approche », adoptée au sein des Communautés européennes en 1985 par le biais d'une Résolution 15 et relayée par le législateur suisse. Il s'est agi, tant en droit européen qu'en droit suisse, de suppri-

12 Bühler Theodor, Die Produktsicherheit als Bestandteil der schweizerischen Rechtsordnung, Zurich et al. 2012 (ci-après : Produktsicherheit), p. 7.

13 Directive 2001/95/CE du Parlement européen et du Conseil du 3 décembre 2001, relative à la sécurité générale des produits, JO L 11 du 15 janvier 2002.

14 Message concernant la loi sur la sécurité des produits (Révision totale de la loi fédérale sur la sécurité d'installations et d'appareils techniques) du 24 juin 2008 (FF 2008 6771) (ci-après : Message LSPro), p. 6772.

15 Résolution du Conseil 85/C 136/01 du 7 mai 1985, concernant une nouvelle approche en matière d'harmonisation technique et de normalisation ; Klindt Thomas, Der «new approach » im Produktrecht des europäischen Binnenmarkts: Vermutungswirkung technischer Normung, EuZW 2002133 ss (ci-après : Der «new approach»), p. 135 . 
mer les autorisations administratives ${ }^{16}$, dès lors que les «producteurs, importateurs, détaillants et autres prestataires [ont] toute latitude pour ne mettre sur le marché que des produits sûrs. En édictant des prescriptions plus strictes, donc en restreignant la part de responsabilité personnelle des producteurs, on risquerait de générer des obstacles techniques au commerce. Par ailleurs, faire contrôler à titre préventif les produits par des organes d'exécution publics requerrait des ressources financières qui ne sont pas disponibles $» 17$.

La négociation des premiers textes d'harmonisation au sein des Communautés européennes ayant toutefois révélé que les mesures adoptées en vertu de la «nouvelle approche » ne suffisaient pas à instaurer la confiance nécessaire entre Etats membres, l' «approche globale » a eu pour vocation de compléter la «nouvelle approche » à la fin des années huitante. L' "approche globale» précise principalement les contours des procédures de certification et d'accréditation ${ }^{18}$ et exerce également une influence en droit suisse. La Confédération a en effet conclu un accord bilatéral avec la Communauté européenne s'agissant de la reconnaissance mutuelle des rapports d'essai, des certificats, des marques et des déclarations de conformité ${ }^{19}$.

16 Klindt, Der «new approach » (n. 15), p. 134 ss ; Message LSPro, FF 20086803 ; LSPro Hess Hans-Joachim, Produktesicherheitsgesetz (PrSG), Berne 2010 (cité : LSPro-Hess), Art. 4 N 15 ss ; Arrêt du Tribunal administratif fédéral C-914/2013 du 6 octobre 2016 consid. 2.1.4 ; Arrêt du Tribunal administratif fédéral C-2016/2014 du 9 décembre 2015 consid. 3.1.

17 Message LSPro (n. 14), p. 6796.

18 Communication de la Commission européenne du 15 juin 1989, JOCE C 267 du 19 octobre 1989; ATF 138 II 134 consid. 4.3.5.

19 Accord entre la Confédération suisse et la Com-
14 Appelé à réformer la «nouvelle approche » et l' "approche globale» au début des années deux mille, le "nouveau cadre législatif » vise l' « introduction d'une politique globale en matière de surveillance du marché ${ }^{20}$. Il se compose de plusieurs règlements ayant pour ambition de réunir l'ensemble des éléments indispensables pour garantir la sécurité des produits et leur conformité aux exigences adoptées en vue de protéger les intérêts publics et le bon fonctionnement du marché unique $^{21}$. L'objectif poursuivi par cette réforme est de tenir compte de l'existence de tous les opérateurs économiques et de leur rôle respectif par rapport au produit mis sur le marché. Elle a conduit à la révision de huit directives européennes dans le contexte de l' " Alignment Package », entré en vigueur en $2016^{22}$.

15 Le droit de l'Union européenne, antérieur ou adopté dans le contexte du "nouveau cadre législatif» et de l' «Alignment Package », exerce une influence en droit suisse, dans la mesure où la législation helvétique résulte d'une reprise autonome du droit européen correspondant ${ }^{23}$, comme c'est le cas pour la LSPro24, ou dans celle où elle repose sur

munauté européenne relatif à la reconnaissance mutuelle en matière d'évaluation de la conformité, conclu le 21 juin 1999 (RS 0.946.526.81).

20 Commission européenne, Communication - Le Guide bleu relatif à la mise en œuvre de la réglementation de l'Union européenne sur les produits 2016, JOCE 2016 C 2721 ss (ci-après : Le Guide), p. 10.

21 Commission, Le Guide(n. 20), p. 10.

22 Commission, Le Guide(n. 20), p. 27.

23 Cf. p. ex. le document publié par le Département fédéral de justice et police "Modification de l'ordonnance sur les substances explosibles (ordonnance sur les explosifs, OExpl), Explications_», p. 2.

24 Bühler, Produktsicherheit (n. 12), p. 132 ss. 
l'exécution d'un accord bilatéral25, à l'instar de certains aspects de la procédure d'évaluation de conformité dans l'ordonnance fédérale sur les équipements sous pression ${ }^{26} \quad$ (ciaprès : OSEP)27. Cette influence est toutefois plus intense en présence d'un tel accord que lorsque le droit suisse résulte d'une reprise autonome. Appelé à interpréter la loi fédérale sur la responsabilité du fait des produits ${ }^{28}$ (ci-après : LRFP), issue d'une reprise de la directive européenne correspondante, le Tribunal fédéral souligne la nuance en ces termes: " au contraire de ce qui vaut dans d'autres matières (cf. ATF 134 III 218 consid. 3.3), il n'existe toutefois aucune obligation pour les tribunaux suisses, lors de l'interprétation de la LRFP, de tenir compte de la jurisprudence européenne en matière de responsabilité du fait des produits. Cela étant, l'intention du législateur d'adapter le droit suisse au droit européen de manière autonome doit être prise en compte et il convient donc d'éviter de contrecarrer l'harmonisation voulue sans qu'il y ait pour cela un bon motif... ${ }^{29}$.

\section{b) De la LSPro à la LETC en passant législation sectorielle}

16 A l'instar de la directive européenne dont elle s'inspire, la LSPro est une loi généraleso, en ce sens qu'elle a pour vocation de s'appliquer à tous les produits définis à

${ }_{25}$ Cf. supra la note 19.

26 Ordonnance fédérale du 25 novembre 2015 sur la sécurité des équipements sous pression (RS 930.114).

27 Cf. les Explications du Département fédéral de l'économie, de la formation et de la recherche DEFR.

28 Loi fédérale du 18 juin 1993 sur la responsabilité du fait des produits (RS 221.112.944).

29 ATF 137 III 226 consid. 2.2.

30 Message LSPro (n. 14), p. 6772. l'art. 2, et non plus uniquement aux installations et appareils techniques, seuls visés par la LSIT31. Elle est assortie de plusieurs ordonnances, comme l'OSPro ou l'ordonnance du Département fédéral de l'économie, de la formation et de la recherche sur l'exécution de la surveillance du marché conformément à la section 5 de l'ordonnance sur la sécurité des produits $^{32}$ (ci-après : Ocomp-OSPro)33. Cette caractéristique est d'ailleurs commune à la LSPro et à LETC. Comme le souligne le Tribunal fédéral, la LETC établit des règles uniformes applicables dans les domaines où la Confédération est compétente pour légiférer, visant à empêcher la création d'entraves techniques au commerce, à les éliminer ou à les réduire (art. 1 al. 1 LETC). Elle contient en particulier des règles concernant la mise sur le marché de produits fabriqués selon des prescriptions techniques étrangères. L'art. 4 al. 5 LETC décrit ainsi ces prescriptions de façon similaire aux art. 4 à 6 LSPro. L'art. 19 LETC prévoit en outre des mesures de surveillance du marché reflétant celles de l'art. 10 LSPro.

17 Ainsi, les objectifs des deux lois s'entremêlent. Comme le souligne le Tribunal fédéral, "sowohl das PrSG als auch das THG, insbesondere in seiner revidierten Form, bezwecken eine Harmonisierung der schweizerischen Produktvorschriften mit denjenigen der EU. (...) Damit soll insbesondere - wie bereits im früheren STEG - der sog. New Approach

31 Arrêt du Tribunal administratif fédéral C-914/2013 du 6 octobre 2016, consid. 2.1.4.

32 Ordonnance du DEFR du 18 juin 2010 sur l'exécution de la surveillance du marché conformément à la section 5 de l'ordonnance sur la sécurité des produits (RS 930.111.5).

33 Bühler, Produktsicherheit (n. 12), p. 10. 
der EU übernommen werden »34. Si dans la LSPro, le législateur a toutefois en point de mire la sécurité des produits sur le marché, dans la LETC, c'est l'élimination des entraves au commerce qui est d'abord visée.

A l'instar de la LSPro (art. 1 al. 3 LSPro), la LETC est une loi-cadre. Elle s'applique ainsi subsidiairement à un autre texte réglant de façon plus spécifique la problématique visée (art. 2 al. 2 LETC). C'est d'ailleurs à l'occasion de l'entrée en vigueur de la LSPro que la LETC a fait l'objet d'une révision, ayant en particulier abouti à l'adoption de l'art. 16a LETC, relatif à la mise sur le marché de produits fabriqués conformément à des prescriptions techniques étrangères. Cette disposition vise seulement la situation où le standard européen n'a pas été repris. Elle ne s'applique donc pas au litige soumis au Tribunal fédéral dans le cas d'espèce, dès lors qu'existent des normes harmonisées entre la Suisse et l'Union européenne pour les produits considérés35.

\section{La sécurité visée}

19 Dans l'arrêt commenté, le Tribunal fédéral commence par rappeler l'exigence de sécurité posée par la LSPro pour la mise sur le marché suisse de produits. L'art. 3 al. 1 LSPro admet uniquement un risque nul ou minime pour la santé et la sécurité36 (2.a). Il appartient par ailleurs au producteur d'évaluer ce risque. Des indi-

34 Arrêt du Tribunal fédéral 2C_75/2016 du 10 avril 2017, publié en partie aux ATF 143 II 518, consid. 5.6.1.

35 Arrêt du Tribunal fédéral 2C_75/2016 du 10 avril 2017, publié en partie aux ATF 143 II 518, consid. 5.4.

36 Arrêt du Tribunal fédéral 2C_75/2016 du 10 avril 2017, publié en partie aux ATF 143 II 518, consid. 5.7. cations peuvent lui être fournies par le biais de normes techniques dont le but est de concrétiser les exigences essentielles auxquelles les produits doivent se conformer pour la mise sur le marché. Le respect de ces normes confère au producteur le bénéfice de la présomption de conformité ancrée à l'art. 5 al. 2 LSPro37. Ce bénéfice a des conséquences pour les organes chargés de la surveillance du marché une fois le produit mis en circulation (2.b).

\section{a) Le risque pour la santé et la sécurité}

20 Le législateur exclut à l'art. 3 al. 1 LSPro une sécurité absolue ${ }^{8}$, puisqu'il admet que des produits présentant un risque minime pour la santé et la sécurité soient mis sur le marché. La notion de risque n'est pas davantage explicitée par la LSPro. On relèvera que les versions allemande et italienne de la loi privilégient le terme de danger (dans la version allemande « nicht oder nur geringfügig gefährden » et dans la version italienne «non espone a pericolo »).

21 Le risque désigne une atteinte à un bien juridiquement protégé susceptible de se réaliser. En matière de sécurité des produits, c'est l'atteinte à la « santé et la sécurité » des utilisateurs et des tiers que le législateur veut éviter. Le bien juridique concerné est ainsi l'intégrité corporelle39. Faut-il comprendre par là que l'atteinte à l'intégrité corporelle doit revêtir une cer-

37 Commission, Le Guide (n. 20), p. 42.

38 Bühler Theodor, La législation sur les risques des produits, progrès pour les consommateurs, nouveaux dangers pour les distributeurs, in : Müller/Carron (édit.), Droit de la consommation et de la distribution, les nouveaux défis, Bâle 2013, p. 39 ss (ci-après : La législation), p. 47.

39 LSPro Hess (n. 16), Art. 3 N 5. 
taine intensité pour que le risque ne soit plus acceptable ou est-ce le degré de probabilité de la survenance d'une telle atteinte qui va déterminer ce seuil ? Par ailleurs, dans quelle mesure le critère de l'utilité d'un produit pour la santé et la sécurité pèse dans la détermination du caractère acceptable du risque ? Une telle conception, soit l'adéquation entre le risque et l'utilité du produit, a par exemple été adoptée pour la mise sur le marché des médicaments ${ }^{40}$. La LSPro ne répond pas à ces questions ${ }^{41}$, comme le relève le Tribunal fédéral dans l'arrêt commenté42. La prévention qu'elle recherche nous incite à opter en faveur d'un seuil d'acceptabilité réduit et à rejeter une application trop vite généralisée $\mathrm{du}$ principe du risque-utilité. Pour le reste, c'est à la lumière des critères énoncés à l'art. 3 LSPro que se détermine le risque acceptable, tout en gardant à l'esprit leur caractère non exhaustif.

Même si l'importateur comme le distributeur, voire même le détaillant peuvent jouer un rôle subsidiaire43, il appartient d'abord au producteur de veiller à la sécurité des produits destinés à être mis sur le marché (art. 3 al. 6 LSPro). Conformément à la "nouvelle approche ", c'est donc au producteur qu'il incombe

40 Büyüksagis Erdem, La relativité de la sécurité du produit : différentes circonstances, différents défauts, différents régimes de responsabilité, RDS 2010 I 29 ss (ci-après : La relativité), p. 43 ; Junod Valérie, La responsabilité de la société pharmaceutique, in : Chappuis/Winiger (édit.), La responsabilité pour l'information fournie à titre professionnel, Journée de la responsabilité civile 2008, Genève et al. 2009, p. 91 ss (ciaprès : La responsabilité), p. 94 ; Werro Franz, La responsabilité civile, $3^{\mathrm{e}}$ éd., Berne 2017, N 673 ss.

41 LSPro Hess (n. 16), Art. 3 N 9.

42 Arrêt du Tribunal fédéral 2C_75/2016 du 10 avril 2017, publié en partie aux ATF 143 II 518, consid. 5.6.3.

43 LSPro Hess (n. 16), Art. 3 N 70. d'évaluer le risque que présente son produit pour la santé et la sécurité des utilisateurs et des tiers. Dans cet exercice, il doit identifier non seulement l'utilisation à laquelle il destine le produit, soit l'utilisation «dans des conditions normales »44, mais également celle qui est « raisonnablement prévisible »45 (art. 3 al. 1 LSPro). Il est ainsi raisonnablement prévisible d'utiliser une cafetière sur une surface mouillée et froide ${ }^{46}$, même s'il ne s'agit pas de l'utilisation à laquelle le fabricant destine l'objet47. Pour déterminer le caractère raisonnablement prévisible d'un produit par rapport à sa destination, il faut également tenir compte du cercle des personnes appelées à entrer en contact avec celui-ci. Il faut en particulier tenir compte du fait que l'utilisateur peut être une personne privée, donc un consommateur (art. 3 al. 3 let. c LSPro), et faire partie d'une tranche de la population particulièrement vulnérable (art. 3 al. 3 let. d LSPro).

23 Un risque spécifique peut en outre engendrer l'adoption de mesures particulières. Un tel risque existe lorsque le produit est dangereux par nature, comme c'est le cas pour les médicaments ${ }^{48}$. Si le risque peut être évité par un certain comportement de l'utilisateur ou d'un tiers, le producteur ou le responsable de la mise sur le marché a le devoir d'en informer les intéressés. Il s'agit en d'autres termes de favoriser l'utilisation à laquelle

44 LSPro Hess,(n. 16), Art. 3 N 12.

45 LSPro Hess (n. 16), Art. 3 N 17, cite en exemple le matelas gonflable d'appoint utilisé comme matériel de natation.

46 ATF 133 III 81.

47 Büyüksagis, La relativité (n. 40), p. 43.

48 Büyüksagis, La relativité (n. 40), p. 44 ; sur le devoir d'information des sociétés pharmaceutiques, cf. Junod, La responsabilité (n. 40), p. 94 ss. 
sont destinés les produits et celle qui est raisonnablement prévisible.

24 Cette information passe d'abord par l'étiquetage et la présentation des produits (art. 3 al. 4 let. a LSPro). Elle peut ensuite passer par l'emballage et les instructions d'assemblage, d'installation et d'entretien des produits (art. 3 al. 4 let. b LSPro), ou encore la mise en garde et les consignes de sécurité (art. 3 al. 4 let. c LSPro), de même que les instructions concernant l'utilisation et l'élimination des produits (art. 3 al. 4 let. d LSPro). La liste n'est toutefois pas exhaustive : le producteur ou le responsable de la mise sur le marché doit en effet communiquer toute autre indication pertinente (art. 3 al. 4 let. e LSPro). Encore faut-il en outre que l'information soit compréhensible, ce qui peut se révéler délicat selon le cercle des personnes visées49. Cette information n'exempte de surcroît pas le producteur ou le responsable de la mise sur le marché de veiller à ce que ses produits soient suffisamment sûrs $5^{\circ}$.

Les indications des art. 3 al. 3 et 4 LSPro ne pallient pas l'absence de disposition légale décrivant la limite entre risque acceptable et risque inacceptable51. Elles contribuent cependant à permettre au producteur de déterminer si son produit correspond aux exigences essentielles de l'art. 3 al. 2 LSPro. Ces « exigences essentielles » sont un concept d'origine euro-

49 Holliger-Hagmann Eugénie, Produktesicherheitsgesetz PrSG, Zurich et al. 2010 (ci-après: Produktesicherheitsgesetz), p. 33 s. ; Büyüksagis, La relativité (n. 40), p. 45.

5o ATF 133 III 81 consid. 3.1; Bühler, La législation (n. 38), p. 49.

51 Arrêt du Tribunal fédéral 2C_75/2016 du 10 avril 2017, publié en partie aux ATF 143 II 518, consid. 5.7 . péenne fonction du danger inhérent à un produit. De telles exigences fixent les objectifs à atteindre ou définissent les dangers à traiter, sans toutefois préciser les solutions techniques pour y parvenir 52 . Les solutions peuvent être apportées par des normes harmonisées, soit des normes européennes adoptées à la suite d'une demande formulée par la Commission européenne à des organisations européennes de normalisation. De telles normes peuvent offrir au producteur le moyen de diminuer ou d'éliminer le risque que présentent certains produits53. Il lui appartient toutefois d'évaluer ce risque et d'identifier les exigences essentielles applicables de manière à choisir les normes harmonisées pertinentes54. En l'absence d'exigences essentielles, le producteur n'aura par ailleurs pas d'autre choix que de démontrer que son produit correspond à l'état des connaissances et de la technique (art. 3 al. 2 LSPro).

26 Comme pour tous les produits visés par l'art. 2 LSPro, les machines doivent en effet, pour leur mise sur le marché, être conformes aux exigences essentielles ou, à défaut de telles exigences, correspondre à l'état des connaissances et de la technique (art. 3 al. 2 LSPro). En vertu de l'art. 4 al. 1 LSPro, c'est au Conseil fédéral qu'il incombe de fixer ces exigences, en tenant compte du droit international pertinent (art. 4 al. 2 LSPro). Pour les machines du type de celles en cause dans l'arrêt commenté, l'autorité a ainsi adopté l'Ordonnance sur la sécurité des machines55 (ci-après: OMach). Comme le rappelle l'art. 1 al. 3 LSPro, ce sont les

$5^{2}$ Commission, Le Guide (n. 20), p. 39.

53 Commission, Le Guide (n. 20), p. 39.

54 Commission, Le Guide (n. 20), p. 42.

55 Ordonnance sur la sécurité des machines du 2 avril 2008 (RS 819.14). 
dispositions de l'OMach qui poursuivent le même but que la LSPro qui s'appliquent à l'exclusion de cette loi.

L'art. 2 OMach exige que les machines au sens de l'art. 1 al. 3 OMach répondent de manière générale aux exigences posées par la Directive 2006/42/CE relative aux machines. Il incombe par ailleurs au SE$\mathrm{CO}$ de déterminer les normes techniques aptes à concrétiser les exigences essentielles en matière de santé et de sécurité telles qu'elles résultent de ce droit (art. 3 OMach). Pour les machines litigieuses, le SECO a désigné la norme technique «EN 474-1 »56. Il s'agit d'une norme européenne harmonisée concrétisant les exigences essentielles mentionnées pour la mise sur le marché. Plusieurs versions de la norme existent cependant, du fait de révisions successives en droit de l'Union européenne. Dans son jugement, le Tribunal administratif fédéral n'avait pas précisé la version de la norme harmonisée pertinente. Dans la documentation fournie par le producteur se trouve une déclaration de conformité à la norme dans sa version du début des années 2000. Ce point n'est toutefois pas celui mis en avant par le DEFR dans son recours, car la dernière version de la norme n'a pas subi de modifications sur le point reproché par le Département au producteur. Le Tribunal fédéral constate donc que les machines litigieuses respectent la norme « EN 474-1 »57.

Le litige porte dès lors sur la question de savoir si, comme l'allèguent la SUVA et le DEFR, une solution technique était envi-

56 Arrêt du Tribunal fédéral 2C_75/2016 du 10 avril 2017, publié en partie aux ATF 143 II 518, consid. 6.1.

57 Arrêt du Tribunal fédéral 2C_75/2016 du 10 avril 2017, publié en partie aux ATF 143 II 518, consid. 6.5 . sageable pour éviter qu'un conducteur de machine croie par erreur à un assemblage correct des accessoires, empêchant la chute d'un objet assemblé de façon incorrecte $^{5}$. Selon le DEFR, le producteur doit dans chaque cas évaluer le risque que présente son produit, nonobstant le respect d'une norme harmonisée. Le Tribunal fédéral lui donne raison, en précisant toutefois que cet examen vise à identifier les lacunes de couverture de la norme, soit les risques non envisagés par celle-ci. Pour le risque pris en compte par la norme, une telle évaluation n'est pas nécessaire59. La Haute Cour examine dès lors si la norme «EN 474-1» prend en compte le risque de verrouillage incomplet et parvient à la conclusion que tel est le $\operatorname{cas}^{60}$. De ce constat, il découle que le producteur peut bénéficier de la présomption de conformité ancrée à l'art. 5 al. 2 LSPro. Autre est la question de savoir si la mesure prévue par la norme réduit suffisamment le risque ${ }^{61}$. Comme exposé ci-après, il appartient aux organismes de surveillance du marché de l'aborder.

\section{b) Les conséquences pour la surveillance du marché}

29 Lorsque le producteur respecte les normes techniques, il peut partir du principe, on l'a dit, que son produit satisfait aux exigences essentielles en matière

58 Arrêt du Tribunal fédéral 2C_75/2016 du 10 avril 2017, publié en partie aux ATF 143 II 518, consid. 6.5.

59 Arrêt du Tribunal fédéral 2C_75/2016 du 10 avril 2017, publié en partie aux ATF 143 II 518, consid. 6.6.

60 Arrêt du Tribunal fédéral 2C_75/2016 du 10 avril 2017, publié en partie aux ATF 143 II 518, consid. 7.

61 Arrêt du Tribunal fédéral 2C_75/2016 du 10 avril 2017, publié en partie aux ATF 143 II 518, consid. $7 \cdot 3$. 
de santé et de sécurité. Cela se vérifie pour les machines litigieuses. Ainsi, l'OMach oblige-t-elle le producteur à évaluer le risque présenté par son produit et lui indique par un renvoi au droit de l'Union européenne les éléments à respecter en vue de remplir les exigences essentielles. Il ne s'agit toutefois là que de principes. Il s'ensuit que la présomption de conformité ne donne au producteur qu'une sécurité juridique relative, en ce sens qu'il est dispensé de prouver la conformité aux exigences essentielles pour ce que couvre la norme harmonisée, s'il démontre s'être conformé à celle-ci. Il incombe alors aux organes de surveillance du marché d'apporter la preuve que le produit ne satisfait pas aux critères de sécurité applicables pour la mise sur le marché.

30 En vertu de l'art. 9 LSPro, c'est au Conseil fédéral qu'il revient d'organiser la surveillance générale du marché et d'en assurer l'exécution, sous réserve d'une réglementation sectorielle sur le sujet. Une telle réglementation existe par exemple pour les produits de construction pour lesquels l'Office fédéral des constructions et de la logistique est à la fois l'autorité de surveillance, d'exécution et de contrôle ${ }^{62}$. Lorsqu'elle repose sur la LSPro, l'organisation de l'exécution des prescriptions de contrôle ressortit au SECO, qui doit agir de concert avec les organes d'exécution (art. 3 al. 1 OSPro). Ces organes sont désignés à l'art. $20 \mathrm{OSPro}^{63}$. Ils revêtent la forme d'institutions publiques, à l'exemple de la SUVA, comme d'institutions privées ${ }^{64}$, tel

62 Art. 29 al. 3 de la loi fédérale du 21 mars 2014 sur les produits de construction (RS 933.0).

63 LSPro Hess (n. 16), Art. 9 N 12.

64 LSPro Hess (n. 16), Art. 9 N 17 ; Von Büren Lucie, Akkreditierte Zertifizierung im gesetzlich geregel- le Bureau de prévention des accidents (ci-après : BPA). Pour certains produits, le contrôle peut être le fait d'entités justifiant de connaissances spécifiques désignées par le Département fédéral de l'économie, de la formation et de la recherche (art. 20 al. 1 let. c OSPro) ${ }^{65}$. Tel est le cas de l'Association suisse d'inspection technique (ASIT) pour les récipients à pression et équipements sous pression ${ }^{66}$, mais également de la SUVA dans le cas qui nous occupe, le DEFR ayant désigné cette institution dans une ordonnance. A noter qu'en vertu de l'art. 23 OSPro, la loi fédérale sur la procédure administrative ${ }^{67}$ s'applique aussi aux entités spécialisées, en leur qualité d'organes de contrôle non soumis au droit public ${ }^{68}$. Cela vaut également lorsque la surveillance est exercée par des organismes privés ${ }^{69}$ L'art. 12 LSPro soumet en particulier les organes d'exécution à une obligation de garder le secret et l'art. 13 LSPro consacre expressément le principe de la protection des données.

31 Une confusion peut résulter de l'utilisation des termes « organes de contrôle » et «organes d'exécution» tant par le législateur ${ }^{\circ}$ que par le Tribunal

ten Bereich, thèse, Berne 2013, p. 142 s. (ciaprès : Akkreditierte Zertifizierung).

65 Cf. l'ordonnance du 18 juin 2010 sur l'exécution de la surveillance du marché conformément à la section 5 de l'ordonnance sur la sécurité des produits (RS 930.111.5).

66 Annexe 1 de l'OComp-OSPro.

67 Loi fédérale du 20 décembre 1968 sur la procédure administrative (RS 172.021).

68 Von Büren, Akkreditierte Zertifizierung (n. 64), p. 76 et la note 349 .

69 Bühler, Produktsicherheit (n. 12), p. 118.

70 Le législateur privilégie les termes d' « organes de contrôle » à l'art. 16 al. 2 OSPro, art. 20 et 22 OSPro, en particulier l'alinéa 5 qui renvoie toutefois à l'art. 10 LSPro où figurent les termes d' « organes d'exécution ». 
fédéral ${ }^{71}$ et le Tribunal administratif fédéral. En réalité, les organes de contrôle effectuent matériellement les contrôles prescrits par la législation en matière de sécurité des produits. En vertu des art. 10 LSPro et 20 al. 1 OSPro, ils doivent veiller à l'application des prescriptions sur la mise sur le marché. L’art. 22 OSPro définit plus précisément leurs tâches ${ }^{72}$. Ils doivent ainsi vérifier, notamment par sondages, le respect des exigences de sécurité auxquels doivent répondre les produits. Ces organes doivent mettre en œuvre des contrôles plus poussés « s’il y a des raisons de penser » que ces prescriptions ne sont pas respectées (art. 22 al. 5 OSPro). Les organes de contrôle sont également des « organes d'exécution », avec et sous la surveillance du SECO, selon l'art. 25 OSPro73.

32 Dans le cas litigieux, la surveillance exercée par la SUVA s'est concrétisée dans le prononcé d'une interdiction de mise sur le marché des machines. L'organe de contrôle a fondé sa décision sur les caractéristiques techniques des produits, sans toutefois entrer dans les détails. Dans sa prise de position devant le Tribunal administratif fédéral, le DEFR a argumenté en ce sens que le verrouillage défectueux ne pouvait pas être évité par des moyens techniques. La question qui se pose est dès lors celle de savoir si ce constat suffit à admettre que le produit ne répond pas

71 Arrêt du Tribunal fédéral 2C_79/2016 du 10 avril 2017 consid. 5.1: organes d'exécution pour désigner la SUVA qui répond à la qualification d'organe de contrôle selon l'art. 20 OSPro;; Arrêt du Tribunal administratif fédéral A_3085/2016, 20 juin 2017 consid. 3.3.6 (organes de contrôle), consid. 3.4.3 (organes d'exécution).

72 Bühler, Produktsicherheit (n. 12), p. 112.

73 Arrêt du Tribunal administratif fédéral C-485/2016 du 17 mai 2017, consid. 5.2.5. aux exigences fixées par le législateur à l'art. 3 LSPro.

33 Pour y répondre, le Tribunal fédéral se concentre sur le risque présenté par les machines litigieuses. Ce risque concerne le verrouillage incorrect des systèmes d'attache rapide car il s'agit d'une utilisation avec laquelle il faut compter et qui est raisonnablement prévisible ${ }^{74}$. Les juges de Mon-Repos précisent que la sécurité doit d'abord résulter de la construction des machines. Elle repose ensuite sur la prise de mesures contre les risques inévitables et elle dépend en dernier lieu de l'information à fournir à l'utilisateur sur les risques éventuels. Ces mesures doivent être prises indépendamment de l'intensité du risque. Même un risque réduit suppose ainsi de s'assurer que la construction de la machine permet d'éviter ou de diminuer le danger, pour autant que cela soit possible.

34 La Directive 2006/42/CE relative aux machines est utile dans ce contexte, puisqu'elle précise que les mesures à prendre dépendent de la gravité de l'atteinte à la santé possible comme de la probabilité de sa survenance. Plus le préjudice susceptible de se produire est important, plus des mesures sévères sont nécessaires. Comme dans le cas d'espèce il est incontesté que l'utilisation incorrecte du système de verrouillage peut engendrer des dommages corporels graves, il existe un risque à juguler. La norme «EN 474-1 » en tient compte, mais seulement pour l'utilisation normale des machines. En cas de manipulations in-

$74 \overline{\text { Arrêt du Tribunal fédéral 2C_75/2016 du } 10 \text { avril }}$ 2017, publié en partie aux ATF 143 II 518, consid 8.3.1. 
correctes raisonnablement prévisibles, elle ne prévoit que des mesures d'ordre organisationnel et aucune prescription au stade de la construction. Or de telles mesures ne doivent entrer en ligne de compte que lorsque des mesures techniques de construction s'avèrent disproportionnées. Tel n'est pas le cas en l'occurrence. En n'exigeant pas que la sécurité soit prise en compte dans la construction de la machine pour diminuer raisonnablement les risques, la norme «EN 474-1 » méconnaît un aspect fondamental des exigences en matière de santé et de sécurité, raison pour laquelle les systèmes d'attache rapide basés sur cette norme ne s'y conforment pas. Il en résulte pour le Tribunal fédéral un renversement de la présomption de sécurité de l'art. 5 al. 2 LSPro75.

35 On peut s'interroger sur l'opportunité de l'utilisation des termes de "renversement de la présomption» («Widerlegung der Konformitätsvermutung »). L'art. 5 al. 2 LSPro contient en effet une présomption légale d'un fait 76 . Elle permet dans le cas particulier de déduire du respect des normes techniques (le faitprémisse) la conformité aux exigences essentielles (le fait à prouver)77. De telles présomptions découlent d'abord de l'art. $8 \mathrm{CC}^{7}$. Elles peuvent toutefois se présenter dans tous les domaines d'application du droit, y compris en droit

75 Arrêt du Tribunal fédéral 2C_75/2016 du 10 avril 2017, publié en partie aux ATF 143 II 518, consid. 8.4.

76 Walter Hans Peter, Berner Kommentar Band/Nr. I/1, Einleitung, Art. 1-9 ZGB Schweizerisches Zivilgesetzbuch, Einleitung und Personenrecht, $\mathrm{N} 403$ ad art. 8 CC.

77 Sur ces questions cf. Steinauer Paul-Henri, Traité de droit privé suisse, Volume II, Le Titre préliminaire du Code civil et Droit des personnes, Tercier (édit.), Bâle 2009 (ci-après : TDP), N 651 ss.

78 Steinauer, TDP (n. 78), N 645 ss. public79. Dès lors que le producteur conserve le fardeau de la preuve du respect des normes techniques, on ne peut à proprement parler de « renversement » du fardeau de la preuve de la conformité $\mathrm{du}$ produit aux exigences essentielles, mais plutôt d'un «allègement » de celuici. Le producteur peut en effet se contenter de prouver qu'il a respecté les normes techniques. Ce fait-prémisse établi, il incombe aux organes de surveillance du marché d'assumer la contre-preuve ou la preuve du contraire. Dans le cas d'espèce, il s'est agi pour la SUVA de démontrer que le produit n'était pas sûr, dès lors que la norme «EN 474.1 » n'appréhendait pas le risque de façon appropriée, dans le cas d'espèce conformément aux prescriptions de la Directive 2006/42/CE relative aux machines ${ }^{80}$.

36 Reste pour le Tribunal fédéral à vérifier que ce constat n'est pas en contradiction avec les engagements que la Suisse a pris avec l'Union européenne, en particulier l'Accord bilatéral entre la Confédération suisse et la Communauté européenne relatif à la reconnaissance mutuelle en matière d'évaluation de la conformité. Les juges de Mon-Repos parviennent à la conclusion que tel n'est pas le cas. Le point de savoir si les normes techniques correspondent aux exigences essentielles en matière de santé et de sécurité ne fait en effet pas l'objet de cet accord, mais ressortit exclusivement à la législation sur la sécurité des produits, soit avant

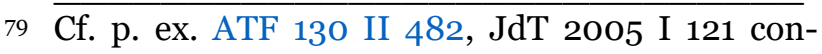
sid. 3.2 et les réf. citées ; Arrêt du Tribunal fédéral 5A_98/2011 du 3 mars 2011 consid. 2.3.

80 Arrêt du Tribunal fédéral 2C_75/2016 du 10 avril 2017, publié en partie aux ATF 143 II 518, consid. 8.3. 
tout à la LSPro ${ }^{81}$. En vertu de l'art. 12 al. 4 de l'Accord en revanche, la Suisse doit informer l'Union européenne de la lacune constatée par rapport à la norme «N 474-1 »82.

37 En tout état de cause, il incombe au producteur d'identifier les mesures de construction aptes à éliminer ou à tout le moins à diminuer le risque. Même si le texte légal (art. 10 al. 2 LSPro) semble indiquer le contraire, la LSPro - comme la LETC - se fondent sur le principe de l' « auto-régulation » régulée, par quoi il faut comprendre le fait que l'Etat pose le cadre au sein duquel la régulation revient aux privés. Il appartient donc au producteur de décider comment, respectivement par quelle mesure de construction raisonnable il convient de parer au défaut. Ce point de vue se justifie à notre avis si l'on garde à l'esprit la «nouvelle approche » et la latitude laissée au producteur dans ce contexte.

38 Une fois la mesure adoptée, le produit peut être à nouveau mis sur le marché, car il ne sera plus identique du point de vue de la sécurité des produits à celui mis originairement en circulation ${ }^{83}$.

\section{Conclusion}

39 Dans cet arrêt rendu en matière de sécurité des machines, le Tribunal fédéral donne un éclairage bienvenu sur le modus operandi dans le domaine, mais éga-

81 Arrêt du Tribunal fédéral 2C_75/2016 du 10 avril 2017, publié en partie aux ATF 143 II 518, consid. 9.

82 Arrêt du Tribunal fédéral 2C_75/2016 du 10 avril 2017, publié en partie aux ATF 143 II 518, consid. 9.4.

83 Arrêt du Tribunal fédéral 2C_75/2016 du 10 avril 2017, publié en partie aux ATF 143 II 518, consid. 10. lement de manière plus générale en droit de la sécurité des produits. Ainsi, lorsqu'existe une norme technique concrétisant les exigences essentielles en matière de santé et de sécurité, le producteur qui la respecte peut se prévaloir de la présomption de conformité sans devoir encore procéder à une évaluation des risques envisagés par la norme. La présomption ne vaut toutefois que pour ces risques.

40 La Haute Cour parle dans ce cas d'un « renversement " du fardeau de la preuve. Il serait toutefois préférable de privilégier le terme d' " allègement » de ce fardeau, en ce sens que le producteur peut, dans le contexte de la surveillance du marché, se limiter à prouver qu'il a respecté les normes techniques, les organes de surveillance du marché devant alors apporter la preuve que ces normes ne tiennent pas compte de façon appropriée des risques que présente le produit.

41 La latitude laissée au producteur par la «nouvelle approche » s'agissant de la manière de se conformer aux exigences essentielles explique la raison pour laquelle le respect des normes techniques ne confère à ce dernier qu'une sécurité juridique relative. Lorsqu'une norme technique ne tient pas correctement compte d'un risque non admis par l'art. 3 al. 1 LSPro, le producteur ne peut pas prétendre avoir respecté les exigences essentielles en matière de santé et de sécurité, même s'il s'est conformé à cette norme.

42 La mise en lumière d'une lacune de la norme aura en général lieu dans le contexte de la surveillance du marché. Le respect de la norme permet en effet au 
producteur de faire l'économie de déterminer si les mesures prévues par celle-ci répondent aux exigences en matière de santé et de sécurité. Tel était en l'occurrence le cas, puisque la norme technique appréhendait certes le risque incriminé, mais de façon inadéquate.

43 S'agissant en revanche du risque dont la norme technique ne tient pas compte, son identification incombe au producteur en vertu de l'art. 3 al. 2 LSPro. En d'autres termes, ce dernier ne peut pas faire l'économie d'évaluer le risque que son produit peut présenter en dehors du cadre fixé par une norme technique et de prendre au besoin les mesures adéquates pour que ce produit présente un risque nul voire minime pour la santé des utilisateurs. Dans le cas contraire, il s'expose au prononcé des mesures prévues à l'art. 10 LSPro. 